PROCEEDINGS OF THE

AMERICAN MATHEMATICAL SOCIETY

Volume 131, Number 5, Pages 1573-1582

S 0002-9939(02)06866-1

Article electronically published on December 16, 2002

\title{
EXISTENCE AND NONEXISTENCE OF GLOBAL SOLUTIONS OF SOME NON-LOCAL DEGENERATE PARABOLIC SYSTEMS
}

\author{
WEIBING DENG, YUXIANG LI, AND CHUNHONG XIE
}

(Communicated by David S. Tartakoff)

\begin{abstract}
This paper establishes a new criterion for global existence and nonexistence of positive solutions of the non-local degenerate parabolic system

$$
\begin{aligned}
& u_{t}=v^{p}\left(\Delta u+a \int_{\Omega} v d x\right), \\
& v_{t}=u^{q}\left(\Delta v+b \int_{\Omega} u d x\right), \quad x \in \Omega, t>0,
\end{aligned}
$$

with homogeneous Dirichlet boundary conditions, where $\Omega \subset \mathbb{R}^{N}$ is a bounded domain with a smooth boundary $\partial \Omega$ and $p, q, a, b$ are positive constants. For all initial data, it is proved that there exists a global positive solution iff $\int_{\Omega} \varphi(x) d x \leq 1 / \sqrt{a b}$, where $\varphi(x)$ is the unique positive solution of the linear elliptic problem $-\Delta \varphi(x)=1, x \in \Omega ; \varphi(x)=0, x \in \partial \Omega$.
\end{abstract}

\section{INTRODUCTION}

In [1, the authors investigate the global existence and nonexistence of positive solutions of the strongly coupled degenerate parabolic system

$$
\begin{aligned}
& u_{t}=v^{p}(\Delta u+a u), \\
& v_{t}=u^{q}(\Delta v+b v), \quad x \in \Omega, t>0,
\end{aligned}
$$

with homogeneous Dirichlet boundary conditions. It is shown that there exists a global positive solution if and only if $\lambda_{1} \geq \min \{a, b\}$, where $\lambda_{1}$ is the first Dirichlet eigenvalue for the Laplacian on $\Omega$.

In this paper, we study a new parabolic system with a non-local source

$$
\begin{aligned}
u_{t} & =v^{p}\left(\Delta u+a \int_{\Omega} v d x\right), & & \\
v_{t} & =u^{q}\left(\Delta v+b \int_{\Omega} u d x\right), & & x \in \Omega, t>0, \\
u(x, t) & =v(x, t)=0, & & x \in \partial \Omega, t>0, \\
u(x, 0) & =u_{0}(x), \quad v(x, 0)=v_{0}(x), & & x \in \Omega,
\end{aligned}
$$

where $\Omega \subset \mathbb{R}^{N}$ is a bounded domain with a smooth boundary $\partial \Omega$ and $p, q, a, b$ are positive constants.

Received by the editors January 8, 2002.

2000 Mathematics Subject Classification. Primary 35K50, 35K55, 35K65.

Key words and phrases. Global existence-nonexistence, degenerate parabolic system, nonlocal. 
Over the past several years, a variety of non-local parabolic equations were studied by many authors (see [2]-10] and references therein). In particular, some authors [8]-[10] studied a class of non-local degenerate parabolic equations which arise in a model of population that communicates through chemical means.

In order to motivate the main result for system (1.2), we recall a classical result of Galaktionov et al. (see [12, 13]) for the system

$$
\begin{aligned}
& u_{t}=\Delta u^{\nu+1}+v^{p}, \\
& v_{t}=\Delta v^{\mu+1}+u^{q}, \quad x \in \Omega, t>0
\end{aligned}
$$

with homogeneous Dirichlet boundary conditions. It is shown that if $p q<$ $(1+\mu)(1+\nu)$, every solution of (1.3) is global, while if $p q>(1+\mu)(1+\nu)$, there are solutions that blow up and others that are global. In the critical case where $p=1+\mu, q=1+\nu$, they proved that:

(1) If $\lambda_{1}>1$, all solutions of (1.3) are global.

(2) If $\lambda_{1}<1$, there are no nontrivial global solutions of (1.3).

Their results show that the first eigenvalue $\lambda_{1}$ plays a crucial role in the critical case $p q=(1+\mu)(1+\nu)$ (see also [14, [15]).

Similar results have also been obtained for the scalar equation

$$
u_{t}=u^{p}(\Delta u+u) \text {. }
$$

It was shown that there exists a unique positive solution which blows up in finite time if $\lambda_{1}<1$ and exists globally if $\lambda_{1} \geq 1$ (see [16]-[18] and the references therein). But, for system (1.2), it seems that $\lambda_{1}$ no longer takes action. Motivated by these results, in this paper we will establish a new criterion for global existence and nonexistence of solutions for system (1.2).

Throughout this paper, the initial values and the boundary $\partial \Omega$ are assumed to satisfy

$$
\begin{aligned}
& \partial \Omega \in C^{2+\alpha}, \\
& u_{0}(x), v_{0}(x) \in C^{1}(\bar{\Omega}), \quad u_{0}(x), v_{0}(x)>0 \text { in } \Omega, \\
& u_{0}(x)=v_{0}(x)=0, \quad \partial u_{0} / \partial n, \partial v_{0} / \partial n<0 \text { on } \partial \Omega .
\end{aligned}
$$

Definition 1.1. A positive solution of the system (1.2) is a vector function $(u, v) \in$ $C\left(\bar{\Omega} \times\left[0, T^{*}\right)\right) \cap C^{2,1}\left(\Omega \times\left(0, T^{*}\right)\right)$, positive in $\Omega \times\left(0, T^{*}\right)$ and satisfying (1.2), where $T^{*}$ is the maximal existence time of the solution. If $T^{*}=\infty$, we say $(u, v)$ is global.

In our considerations a crucial role is played by

$$
\mu=\int_{\Omega} \varphi(x) d x
$$

where $\varphi(x)$ is the unique positive solution of the following linear elliptic problem

$$
-\Delta \varphi(x)=1, x \in \Omega ; \varphi(x)=0, x \in \partial \Omega .
$$

Then, let us state our main result.

Theorem 1.2. Assume that (H1) holds. Then there exists a global positive solution of (1.2) iff $\mu^{2} \leq 1 /(a b)$. 
We are also interested in another non-local degenerate parabolic system, which is of the form

$$
\begin{aligned}
& u_{t}=v^{p}\left(\Delta u+a \int_{\Omega} u d x\right), \\
& v_{t}=u^{q}\left(\Delta v+b \int_{\Omega} v d x\right), \quad x \in \Omega, t>0,
\end{aligned}
$$

with similar initial-boundary conditions as in (1.2). For system (1.6), we get a different criterion as follows.

Theorem 1.3. Assume that (H1) holds. Then there exists a global positive solution of (1.6) iff $1 / \mu \geq \min \{a, b\}$.

The result shows that for system (1.6), it is not $\lambda_{1}$ but $1 / \mu$ that plays a crucial role. We will not discuss (1.6) in detail since it can be easily proved by combining the present arguments with those in [1].

Remark 1.4. Combining the arguments in [1] and in the present paper, we can show that $\lambda_{1}^{2} \geq a b$ is the critical condition of system

$$
u_{t}=v^{p}(\Delta u+a v), \quad v_{t}=u^{q}(\Delta v+b u) .
$$

We will not give the proof here, since this paper is concerned about the non-local problem.

This paper is organized as follows. Section 2 establishes the local theory. Section 3 gives the proof of the main result.

\section{LOCAL EXISTENCE}

Set $Q_{T}=\Omega \times(0, T], S_{T}=\partial \Omega \times(0, T]$ for $0<T<\infty$. We first give a maximum principle for non-local systems, of which the proof is standard, and omit its proof.

Proposition 2.1. Suppose that $w_{1}(x, t), w_{2}(x, t) \in C\left(\overline{Q_{T}}\right) \cap C^{2,1}\left(Q_{T}\right)$ satisfy

$$
\begin{array}{ll}
w_{1 t}-d_{1} \Delta w_{1} \geq c_{11} w_{1}+c_{12} w_{2}+c_{13} w_{1} w_{2} & \\
\quad+c_{14} \int_{\Omega} c_{15} w_{1}(x, t) d x+c_{16} \int_{\Omega} c_{17} w_{2}(x, t) d x, & \\
w_{2 t}-d_{2} \Delta w_{2} \geq c_{21} w_{1}+c_{22} w_{2}+c_{23} w_{1} w_{2} & \\
\quad+c_{24} \int_{\Omega} c_{25} w_{1}(x, t) d x+c_{26} \int_{\Omega} c_{27} w_{2}(x, t) d x, & \\
w_{1}(x, t) \geq 0, w_{2}(x, t) \geq 0, & (x, t) \in Q_{T}, \\
w_{1}(x, 0) \geq 0, w_{2}(x, 0) \geq 0, & x \in \Omega,
\end{array}
$$

where $d_{i}(x, t), c_{i j}(x, t)(i=1,2 ; j=1, \ldots, 7)$ are bounded functions and

$$
d_{1}, d_{2}, c_{12}, c_{21}, c_{1 j}, c_{2 j} \geq 0, j=4, \ldots, 7 \text { in } Q_{T} .
$$

Then $w_{j}(x, t) \geq 0$ on $\overline{Q_{T}}$.

Proposition 2.2. Let $(\tilde{u}, \tilde{v}) \in C\left(\overline{Q_{T}}\right) \cap C^{2,1}\left(Q_{T}\right)$ and $(\bar{u}, \bar{v}) \in C\left(\overline{Q_{T}}\right) \cap C^{2,1}\left(Q_{T}\right)$ be a nonnegative subsolution and a nonnegative supersolution of (1.2), respectively. Assume that $(\bar{u}, \bar{v}) \geq \delta>0$ and either

$$
\Delta \tilde{u}+a \int_{\Omega} \tilde{v} d x \geq 0, \quad \Delta \tilde{v}+b \int_{\Omega} \tilde{u} d x \geq 0
$$


or

$$
\Delta \bar{u}+a \int_{\Omega} \bar{v} d x \geq 0, \quad \Delta \bar{v}+b \int_{\Omega} \bar{u} d x \geq 0
$$

hold. Then $(\tilde{u}, \tilde{v}) \leq(\bar{u}, \bar{v})$ on $\overline{Q_{T}}$ if $\left(\tilde{u}_{0}, \tilde{v}_{0}\right) \leq\left(\bar{u}_{0}, \bar{v}_{0}\right)$.

Proof. This proposition is a direct consequence of Proposition 2.1

Next, in this section, we will give the local existence of the solution for system (1.2) by the same method utilized in 11. For system (1.2) we introduce, for $n=$ $1,2, \ldots$, the following regularized system:

$$
\begin{aligned}
u_{n t} & =v_{n}^{p}\left(\Delta u_{n}+a \int_{\Omega} v_{n} d x\right), & & \\
v_{n t} & =u_{n}^{q}\left(\Delta v_{n}+b \int_{\Omega} u_{n} d x\right), & & x \in \Omega, t>0, \\
u_{n}(x, t) & =v_{n}(x, t)=1 / n, & & x \in \partial \Omega, t>0, \\
u_{n}(x, 0) & =u_{0}(x)+1 / n, \quad v_{n}(x, 0)=v_{0}(x)+1 / n, & & x \in \Omega .
\end{aligned}
$$

By a similar discussion as in (7), under (H1), we can show that (2.3) has a classical solution $\left(u_{n}, v_{n}\right)$ with $u_{n}, v_{n} \geq 1 / n$, defined on $\bar{\Omega} \times\left[0, T_{n}^{*}\right)$, where $T_{n}^{*}$ is the maximal existence time.

Now we construct a uniform upper bound for $\left(u_{n}, v_{n}\right)$. Consider the ordinary differential equation

$$
\begin{aligned}
H^{\prime}(t) & =\hat{a}(H(t))^{\hat{p}}, \\
H(0) & =\max \left\{\max _{x \in \bar{\Omega}} u_{0}(x)+1, \max _{x \in \bar{\Omega}} v_{0}(x)+1\right\},
\end{aligned}
$$

where $\hat{a}=\max \{a|\Omega|, b|\Omega|\}, \hat{p}=\max \{p+1, q+1\}$. Obviously, there exists $T_{0}>0$ such that (2.4) has a non-decreasing solution $H(t)>0$ on $\left[0, T_{0}\right]$; namely, $0<$ $H(0) \leq H(t) \leq H\left(T_{0}\right)<\infty$. Using Proposition 2.2 for system (2.3), we obtain the following lemma.

Lemma 2.3. There exist $T_{0}$ and an a priori bound $H(t)$ depending only on $u_{0}, v_{0}, \hat{a}$ and $\hat{p}$ such that for all $n \geq 1$ the solution of (2.3) satisfies $u_{n}, v_{n} \leq H(t)$ on $\overline{Q_{T_{0}}}$.

Denote by $\lambda_{1}>0$ and $\phi(x)$ the first eigenvalue and the corresponding eigenfunction of the following eigenvalue problem

$$
-\Delta \phi(x)=\lambda \phi(x), x \in \Omega ; \phi(x)=0, x \in \partial \Omega .
$$

It is well known that $\phi(x)$ may be normalized as $\phi(x)>0$ in $\Omega$ and $\max _{\Omega} \phi(x)=1$. Thus, by Proposition 2.1, we have

Lemma 2.4. Let $h(x, t)=k e^{-\rho t} \phi(x)$, where $k$ is small such that $u_{0}, v_{0} \geq k \phi(x)$ and $\rho=\max \left\{\lambda_{1}\left(H\left(T_{0}\right)\right)^{p}, \lambda_{1}\left(H\left(T_{0}\right)\right)^{q}\right\}$. Then for all $n \geq 1$, it holds that $u_{n}, v_{n} \geq$ $h(x, t)$ in $\overline{Q_{T_{0}}}$.

In proving there exists a positive solution of (1.2), we still need the following regularity lemma, whose proof is similar to [1, Lemma 2.3].

Lemma 2.5. $u_{n}, v_{n} \in V_{2}^{1,0}\left(Q_{T_{0}}\right)($ see [19, p. 6]). 
Then by the so-called extension method (for details see [1), we have that there exists a subsequence $\left\{n_{i}\right\}$ of $\{n\}$ such that

$$
\lim _{i \rightarrow \infty}\left(u_{n_{i}}, v_{n_{i}}\right)=(u, v) \quad \text { in } \quad C^{2,1}\left(Q_{T_{0}}\right) .
$$

Similarly, we can show that $u, v$ are continuous at any point $(y, t), y \in \partial \Omega$ and $u(y, t)=0$ (see [16], 20]), and continuous up to $\{t=0\}$ (see [21], 22]).

Let $T^{*}$ be the supremum over $T_{0}$ for which $(u, v)$ exists on $\left(0, T_{0}\right)$. Thus, we have

Theorem 2.6. Assume that (H1) holds. Then there exists a positive solution of (1.2) on $\left(0, T^{*}\right)$. Moreover, if $T^{*}<\infty$, then

$$
\limsup _{t \rightarrow T^{*}}\|u(x, t)\|_{L^{\infty}}=+\infty \quad \text { or } \quad \limsup _{t \rightarrow T^{*}}\|v(x, t)\|_{L^{\infty}}=+\infty .
$$

Remark 2.7. Obviously, all discussions of this section are applicable to system (1.6).

\section{Proof of the MAIN RESUlt}

In order to prove the main result, we give an auxiliary lemma first. Let $G$ be a bounded smooth domain of $\mathbb{R}^{N}$. Consider the problem

$$
\begin{aligned}
w_{t} & =d w^{r}\left(\Delta w+a_{0} \int_{G} w d x\right), & & x \in G, t>0, \\
w(x, t) & =c, & & x \in \partial G, t \geq 0, \\
w(x, 0) & =c, & & x \in G,
\end{aligned}
$$

where $0<r<1$ and $a_{0}, c, d$ are positive constants. By the standard method (see [7], [10]), it follows that (3.1) has a unique classical solution $w(x, t)$ and $w(x, t) \geq c$. Denote by $\varphi_{0}(x)$ the unique positive solution of the linear elliptic problem

$$
-\Delta \varphi_{0}(x)=1, \quad x \in G ; \quad \varphi_{0}(x)=0, \quad x \in \partial G .
$$

Set $\mu_{0}=\int_{G} \varphi_{0}(x) d x$. Thus, we have

Lemma 3.1. If $\mu_{0}>1 / a_{0}$, then the positive solution of (3.1) blows up in finite time.

Proof. Set $F(t)=\int_{G} w^{1-r} \varphi_{0} d x ;$ then

$$
\begin{aligned}
\frac{1}{1-r} F^{\prime}(t) & =d\left(\int_{G} \Delta w \varphi_{0} d x+a_{0} \int_{G} w d x \int_{G} \varphi_{0} d x\right) \\
& \geq d\left(a_{0} \mu_{0}-1\right) \int_{G} w d x \\
& \geq d\left(a_{0} \mu_{0}-1\right)\left(\int_{G} w \varphi_{0} d x\right) / M,
\end{aligned}
$$

where $M=\max _{x \in \bar{G}}\left\{\varphi_{0}(x)\right\}$. Letting $z=w^{1-r}$ in (3.2) yields

$$
\int_{G} z_{t}(x, t) \varphi_{0} d x \geq d(1-r)\left(a_{0} \mu_{0}-1\right)\left(\int_{G} z^{1 /(1-r)} \varphi_{0} d x\right) / M .
$$

Since $\frac{1}{1-r}>1$, by the Jensen inequality, it follows that

$$
\int_{G} z_{t}(x, t) \varphi_{0} d x \geq d(1-r)\left(a_{0} \mu_{0}-1\right)\left(\mu_{0}\right)^{-r /(1-r)}\left(\int_{G} z \varphi_{0} d x\right)^{1 /(1-r)} / M .
$$


That is,

$$
F^{\prime}(t) \geq C_{0}(F(t))^{1 /(1-r)}
$$

where $C_{0}=d(1-r)\left(a_{0} \mu_{0}-1\right)\left(\mu_{0}\right)^{-r /(1-r)} / M>0$. In view of $1 /(1-r)>1$ and $F(0)>0$, it follows that there exists $T<\infty$ such that $\lim _{t \rightarrow T} F(t)=+\infty$, and hence $w(x, t)$ blows up in finite time.

Lemma 3.2. Assume that (H1) holds. Then there exist positive constants $k_{1}, k_{2}$ such that $u(x, t) \geq k_{1} \varphi, v(x, t) \geq k_{2} \varphi$ for $(x, t) \in \bar{\Omega} \times\left[0, T^{*}\right)$ if $\mu^{2} \geq 1 /(a b)$.

Proof. From (H1), since $\mu^{2} \geq 1 /(a b)$ we see that there exist positive constants $k_{1}$ and $k_{2}$ such that

$$
u_{0}(x) \geq k_{1} \varphi(x), \quad v_{0}(x) \geq k_{2} \varphi(x), \quad x \in \bar{\Omega},
$$

and

$$
a \mu \geq k_{1} / k_{2} \geq 1 /(b \mu) .
$$

Let $w(x, t)=u(x, t)-k_{1} \varphi(x), s(x, t)=v(x, t)-k_{2} \varphi(x)$. Then we obtain, by (3.4), for any $T \in\left(0, T^{*}\right)$,

$$
\begin{aligned}
& w_{t}=u_{t}=v^{p}\left(\Delta u+a \int_{\Omega} v d x\right) \\
& =v^{p}\left(\Delta w+a \int_{\Omega} s d x\right)+v^{p}\left(-k_{1}+a k_{2} \mu\right) \\
& \geq v^{p}\left(\Delta w+a \int_{\Omega} s d x\right), \\
& s_{t} \geq u^{q}\left(\Delta s+b \int_{\Omega} w d x\right), \quad x \in \Omega, 0<t \leq T, \\
& w(x, t)=s(x, t)=0, \quad x \in \partial \Omega, 0<t \leq T .
\end{aligned}
$$

By Proposition 2.1, it follows from (3.3) and (3.5) that $w \geq 0, s \geq 0$ and hence $u \geq k_{1} \varphi, v \geq k_{2} \varphi$ on $\bar{\Omega} \times[0, T]$. The arbitrariness of $T$ shows that the result holds.

Lemma 3.3. Assume that (H1) holds. Then no global solution of (1.2) exists if $\mu^{2}>1 /(a b)$.

Proof. Denote by $\varphi_{1}(x)$ the unique positive solution of the linear elliptic problem

$$
-\Delta \varphi_{1}(x)=1, \quad x \in \Omega_{1} ; \quad \varphi_{1}(x)=0, \quad x \in \partial \Omega_{1} .
$$

Here $\Omega_{1} \subset \subset \Omega$. Since the function $U:=\varphi-\varphi_{1} \geq 0$ is harmonic in $\Omega_{1}$ and satisfies $U \leq \varphi$ on $\partial \Omega_{1}$, we have $\left\|\varphi-\varphi_{1}\right\|_{\infty} \leq\|\varphi\|_{L^{\infty}\left(\partial \Omega_{1}\right)}$ by the maximum principle. By the continuity of $\varphi$ it follows that $\left\|\varphi-\varphi_{1}\right\|_{\infty} \rightarrow 0$, as $\operatorname{dist}\left(\partial \Omega_{1}, \partial \Omega\right) \rightarrow 0$. Let $\mu_{1}=\int_{\Omega_{1}} \varphi_{1}(x) d x$. The above discussion implies, in particular, $\mu_{1} \rightarrow \mu$, as $\operatorname{dist}\left(\partial \Omega_{1}, \partial \Omega\right) \rightarrow 0$.

Therefore, in view of $\mu^{2}>1 /(a b)$, we can choose a smooth sub-domain $\Omega_{1} \subset \subset \Omega$ such that $\mu_{1}^{2}>1 /(a b)$. Denote

$$
\delta=\frac{1}{2} \min \left\{k_{1} \underset{\overline{\Omega_{1}}}{\min } \varphi, k_{2} \underset{\overline{\Omega_{1}}}{\min } \varphi\right\} .
$$

Then $\delta>0$ and

$$
u(x, t) \geq 2 \delta, \quad v(x, t) \geq 2 \delta, \quad \forall(x, t) \in \overline{\Omega_{1}} \times\left[0, T^{*}\right),
$$


by Lemma 3.2. Then $(u, v)$ in $\Omega_{1} \times\left(0, T^{*}\right)$ satisfies

$$
\begin{aligned}
u_{t} & =v^{p}\left(\Delta u+a \int_{\Omega} v d x\right) & & \\
& \geq v^{p}\left(\Delta u+a \int_{\Omega_{1}} v d x\right), & & \\
v_{t} & \geq u^{q}\left(\Delta v+b \int_{\Omega_{1}} u d x\right), & & x \in \Omega_{1}, t \in\left(0, T^{*}\right), \\
u(x, t) & \geq 2 \delta, \quad v(x, t) \geq 2 \delta, & & x \in \partial \Omega_{1}, t \in\left(0, T^{*}\right), \\
u(x, 0) & \geq 2 \delta, \quad v(x, 0) \geq 2 \delta, & & x \in \Omega_{1} .
\end{aligned}
$$

Now, we consider the system

$$
\begin{aligned}
\underline{u}_{t} & =\underline{v}^{p}\left(\Delta \underline{u}+a \int_{\Omega_{1}} \underline{v} d x\right), & & \\
\underline{v}_{t} & =\underline{u}^{q}\left(\Delta \underline{v}+b \int_{\Omega_{1}} \underline{u} d x\right), & & x \in \Omega_{1}, t>0, \\
\underline{u}(x, t) & =f(t), \underline{v}(x, t)=g(t), & & x \in \partial \Omega_{1}, t>0, \\
\underline{u}(x, 0) & =\underline{v}(x, 0)=\delta, & & x \in \Omega_{1},
\end{aligned}
$$

where $f(t), g(t)$ satisfy

$$
\begin{aligned}
& f(t), g(t) \in C^{\infty}([0, \infty)), f^{\prime}(t), g^{\prime}(t)>0, f(t), g(t) \leq 2 \delta \\
& f(0)=g(0)=\delta, f^{\prime}(0)=a\left|\Omega_{1}\right| \delta^{p+1}, g^{\prime}(0)=b\left|\Omega_{1}\right| \delta^{q+1}
\end{aligned}
$$

A similar discussion as in 7 shows that there exists a unique classical solution $(\underline{u}, \underline{v}) \in C^{2+\beta, 1+\beta / 2}\left(\overline{\Omega_{1}} \times\left[0, T_{1}\right)\right)$ for some $\beta \in(0,1)$, where $T_{1}$ is the maximal existence time, and

$$
\underline{u}, \underline{v} \geq \delta \quad \text { in } \overline{\Omega_{1}} \times\left[0, T_{1}\right) .
$$

Since the initial data is a subsolution of (3.7), we have $\underline{u}_{t}, \underline{v}_{t} \geq 0$ in $\overline{\Omega_{1}} \times\left[0, T_{1}\right.$ ) and hence

$$
\Delta \underline{u}+a \int_{\Omega_{1}} \underline{v} d x \geq 0, \quad \Delta \underline{v}+b \int_{\Omega_{1}} \underline{u} d x \geq 0 \text { in } \overline{\Omega_{1}} \times\left[0, T_{1}\right) .
$$

Thus from Proposition 2.2. we have $T_{1} \geq T^{*}$ and

$$
u(x, t) \geq \underline{u}(x, t), \quad v(x, t) \geq \underline{v}(x, t) \text { in } \overline{\Omega_{1}} \times\left[0, T^{*}\right) .
$$

Therefore, it suffices to show that $(\underline{u}, \underline{v})$ blows up in finite time, because if so, its upper bound $(u, v)$ does exist up to a finite time $T^{*}$.

By (3.8) and (3.9), we have

$$
\begin{aligned}
& \underline{u}_{t} \geq \delta^{p-r} \underline{v}^{r}\left(\Delta \underline{u}+a \int_{\Omega_{1}} \underline{v} d x\right), \\
& \underline{v}_{t} \geq \delta^{q-r} \underline{u}^{r}\left(\Delta \underline{v}+b \int_{\Omega_{1}} \underline{u} d x\right) \quad \text { in } \quad \Omega_{1} \times\left(0, T_{1}\right)
\end{aligned}
$$

with the corresponding initial and boundary conditions and $0<r<1$.

By use of $\mu_{1}^{2}>1 /(a b)$, there exist positive constants $l_{1}, l_{2}$ with $l_{1}, l_{2}>1$, and $l$ such that

$$
a \mu_{1}>\frac{l_{1}}{l_{2}}>\frac{1}{b \mu_{1}}, \mu_{1}>\frac{1}{l}>\frac{l_{1}}{a l_{2}}, \mu_{1}>\frac{1}{l}>\frac{l_{2}}{b l_{1}} .
$$


Choose

$$
d=\min \left\{\delta^{p-r}, \delta^{q-r}\right\}, \quad \gamma=\min \left\{1 / l_{1}, 1 / l_{2}\right\} .
$$

Denote by $z(x, t)$ the unique positive solution of the problem

$$
\begin{aligned}
z_{t} & =d z^{r}\left(\Delta z+l \int_{\Omega_{1}} z d x\right), & & x \in \Omega_{1}, t>0, \\
z(x, t) & =\gamma \delta, & & x \in \partial \Omega_{1}, t \geq 0, \\
z(x, 0) & =\gamma \delta, & & x \in \Omega_{1},
\end{aligned}
$$

where $l, d, \gamma$ satisfy (3.11) and (3.12). By Lemma 3.1, it follows that $z(x, t)$ blows up in finite time $T_{0}<\infty$. Moreover, $z_{t} \geq 0$, i.e., $\Delta z+l \int_{\Omega_{1}} z d x \geq 0$, since the initial data is a subsolution of (3.13). Let

$$
w(x, t)=l_{1} z(x, t), \quad s(x, t)=l_{2} z(x, t) .
$$

Thus, from (3.11) $-($ (3.13) $)$ and $l_{1}, l_{2}>1$, we have

$$
\begin{gathered}
w_{t}-\delta^{p-r} s^{r}\left(\Delta w+a \int_{\Omega_{1}} s d x\right)=l_{1} d z^{r}\left(\Delta z+l \int_{\Omega_{1}} z d x\right) \\
-l_{1} \delta^{p-r}\left(l_{2} z\right)^{r}\left(\Delta z+\left(a l_{2} / l_{1}\right) \int_{\Omega_{1}} z d x\right) \leq 0, \\
s_{t}-\delta^{q-r} w^{r}\left(\Delta s+b \int_{\Omega_{1}} w d x\right) \leq 0, \quad x \in \Omega_{1}, 0<t<T_{0}, \\
w(x, t)=l_{1} \gamma \delta \leq \delta, s(x, t)=l_{2} \gamma \delta \leq \delta, \quad x \in \partial \Omega_{1}, 0 \leq t<T_{0}, \\
w(x, 0)=l_{1} \gamma \delta \leq \delta, s(x, 0)=l_{2} \gamma \delta \leq \delta, \quad x \in \Omega_{1} .
\end{gathered}
$$

By use of Proposition 2.2, it follows from (3.8), (3.10), (3.14) and $\Delta z+l \int_{\Omega_{1}} z d x \geq 0$ that

$$
(\underline{u}, \underline{v}) \geq\left(l_{1} z, l_{2} z\right) \text { in } \Omega_{1} \times\left(0, T_{1}\right) .
$$

Hence $(\underline{u}, \underline{v})$ blows up in finite time since $z(x, t)$ does. Therefore, $(u, v)$ exists no later than $T_{0}<\infty$. This completes the proof.

Lemma 3.4. Assume that (H1) holds. Then the positive solution $(u, v)$ of (1.2) defined by (2.5) is global if $\mu^{2} \leq 1 /(a b)$.

Proof. Applying $\mu^{2} \leq 1 /(a b)$ and (H1) we see that there exist large positive constants $K_{1}$ and $K_{2}$ such that

$$
a \mu \leq K_{1} / K_{2} \leq 1 /(b \mu)
$$

and

$$
u_{0}(x) \leq K_{1} \varphi(x), \quad v_{0}(x) \leq K_{2} \varphi(x), \quad \forall x \in \bar{\Omega} .
$$


Let $W(x, t)=K_{1} \varphi(x)-u(x, t), S(x, t)=K_{2} \varphi(x)-v(x, t)$. Then, from (3.15), we obtain, for any $T \in\left(0, T^{*}\right)$,

$$
\begin{aligned}
& W_{t}=-u_{t}=-v^{p}\left(\Delta u+a \int_{\Omega} v d x\right) \\
& =v^{p}\left(\Delta W+a \int_{\Omega} S d x\right)+v^{p}\left(K_{1}-a K_{2} \mu\right) \\
& \geq v^{p}\left(\Delta W+a \int_{\Omega} S d x\right), \\
& S_{t} \geq u^{q}\left(\Delta S+b \int_{\Omega} W d x\right), \quad x \in \Omega, 0<t \leq T, \\
& W(x, t)=S(x, t)=0, \quad x \in \partial \Omega, 0<t \leq T .
\end{aligned}
$$

By Proposition 2.1 it follows from (3.16) and (3.17) that $W \geq 0, S \geq 0$ and hence $u \leq K_{1} \varphi, v \leq K_{2} \varphi$ on $\bar{\Omega} \times[0, T]$. The arbitrariness of $T$ shows that $u \leq K_{1} \varphi, v \leq$ $K_{2} \varphi$ on $\bar{\Omega} \times\left[0, T^{*}\right)$. Therefore, the solution $(u, v)$ of (1.2) defined by (2.5) exists globally.

From Lemma 3.3 and Lemma 3.4 , it follows that Theorem 1.2 holds.

Remark 3.5. From Lemma 3.2 and Lemma 3.4, we have that if $\mu^{2}=1 /(a b)$, there exist positive constants $k_{1}, k_{2}, K_{1}$ and $K_{2}$ such that $k_{1} \varphi \leq u(x, t) \leq K_{1} \varphi, k_{2} \varphi \leq$ $u(x, t) \leq K_{2} \varphi$ for $x \in \bar{\Omega}$ and $t>0$.

Remark 3.6. Theorem 1.3 for system (1.6) can be proved by combining the present arguments (for system (1.2)) with those in 1] (for system (1.1)).

\section{ACKNOWLEDGMENTS}

The authors would like to thank the referee for the careful reading of this paper and for valuable suggestions; especially for providing the partial proof of Lemma 3.3 .

\section{REFERENCES}

1. Y.X. Li, W.B. Deng, and C.H. Xie, Global existence and nonexistence for degenerate parabolic systems, Proc. Amer. Math. Soc., 130(2002), 3661-3670.

2. J.W. Bebernes and A. Bressan, Thermal behavior for a confined reactive gas, J. Differential Equations, 44(1982), 118-133. MR 83k:45021

3. C.V. Pao, Blowing-up of solution for a nonlocal reaction-diffusion problem in combustion theory, J. Math. Anal. Appl., 166(1992), 591-600. MR 93c:35073

4. J.M. Chadam, A. Peirce and H.M. Yin, The blowup property of solutions to some diffusion equations with localized nonlinear reactions, J. Math. Anal. Appl., 169(1992), 313-328. MR 93h:35092

5. K. Deng, M.K. Kwong and H.A. Levine, The influence of nonlocal nonlinearities on the long time behavior of solutions of Burger's equation, Quart. Appl. Math., 50(1992), 173-200. MR 92k:35241

6. V.A. Galaktionov and H.A. Levine, A general approach to critical Fujita exponents in nonlinear parabolic problems, Nonlinear Anal., 34(1998), 1005-1027. MR 99f:35079

7. Ph. Souplet, Blow-up in nonlocal reaction-diffusion equations, SIAM J. Math. Anal., 29(1998), 1301-1334. MR 99h:35104

8. J. Furter and M. Grinfeld, Local vs. nonlocal interactions in population dynamics, J. Math. Biol., 27(1989), 65-80. MR 90g:92061

9. J.R. Anderson and K. Deng, Global existence for degenerate parabolic equations with a nonlocal forcing, Math. Methods Appl. Sci., 20(1997), 1069-1087. MR 98f:35083 
10. W.B. Deng, Z.W. Duan and C.H. Xie, The blow-up rate for a degenerate parabolic equation with a non-local source, J. Math. Anal. Appl., 264(2)(2001), 577-597. MR 2002i:35107]

11. J.R. Anderson, Local existence and uniqueness of solutions of degenerate parabolic equations, Comm. Partial Differential Equations, 16(1991), 105-143. MR 92d:35163

12. V.A. Galaktionov, S.P. Kurdyumov and A.A. Samarskii, A parabolic system of quasilinear equations I, Differential Equations, 19(1983), 1558-1571.

13. V.A. Galaktionov, S.P. Kurdyumov and A.A. Samarskii, A parabolic system of quasilinear equations II, Differential Equations, 21(1985), 1049-1062.

14. H.A. Levine, The role of critical exponents in blowup theorems, SIAM Rev., 32(1990), 262288. MR 91j:35135

15. A.A. Samarskii, V.A. Galaktionov, S.P. Kurdyumov and A.P. Mikhailov, Blow-up in quasilinear parabolic equations, Walter de Gruyter, Berlin/New York, 1995. MR 96b:35003

16. M. Wiegner, Blow-up for solutions of some degenerate parabolic equations, Differential and Integral Equations, 7(1994), 1641-1647. MR 95b:35120

17. M. Wiegner, A degenerate diffusion equation with a nonlinear source term, Nonlinear Anal., 28(12)(1997), 1977-1995. MR 97m:35152

18. S. Wang, M.X. Wang and C.H. Xie, A nonlinear degenerate diffusion equation not in divergence form, Z. Angew Math. Phys. 51(2000), 149-159. MR 2001a:35101

19. O. A. Ladyženskaja, V.A. Solonnikov and N.N. Ural'ceva, Linear and quasilinear equations of parabolic type, Amer. Math. Soc., Providence, 1967. MR 39:3159b

20. A. Friedman and B. McLeod, Blow-up of solutions of nonlinear degenerate parabolic equations, Arch. Ration. Mech. Anal., 96(1987), 55-80. MR 87j:35051

21. E. DiBenedetto, Degenerate parabolic equations, Springer-Verlag, New York, 1993. MR 94h:35130

22. E. DiBenedetto, Continuity of weak solutions to a general porous medium equation, Indiana Univ. Math. J., 32(1983), 83-118. MR 85c:35010

Department of Mathematics, Nanjing University, Nanjing 210093, People's Republic OF CHINA

E-mail address: wbdeng@nju.edu.cn

Department of Mathematics, Nanjing University, Nanjing 210093, People's Republic OF CHINA

E-mail address: lieyuxiang@yahoo.com.cn

Department of Mathematics, Nanjing University, Nanjing 210093, People's Republic OF CHINA 